Research Paper

\title{
Preoperative Albumin-Bilirubin Score for Postoperative Solitary Hepatocellular Carcinoma within the Milan Criteria and Child-Pugh A Cirrhosis
}

\author{
Zhao-Ru Dong ${ }^{1,2^{*} \bowtie}$, Jie Zou ${ }^{3 *}$, Dong Sun ${ }^{2}$, Guo-Ming Shi ${ }^{*}$, Ai-Wu Ke ${ }^{1}$, Jia-Bin Cai ${ }^{1}$, Hui-Chuan Sun ${ }^{1}$, \\ Shuang-Jian Qiu1, Tao Li ${ }^{2}$, Jian Zhou ${ }^{1,4}$, Xu-Ting Zhi ${ }^{2}$, Jia Fan ${ }^{1}{ }^{\bowtie}$ \\ 1. Liver Cancer Institute and Zhongshan Hospital, Fudan University, Shanghai 200032, China; \\ 2. Department of General Surgery, Qilu Hospital, Shandong University, Jinan 250012, China; \\ 3. Department of Geriatrics, Qilu Hospital, Shandong University, Jinan, Shandong 250012, China. \\ 4. Cancer Center, Institutes of Biomedical Sciences, Fudan University, Shanghai 200031, China \\ *These authors contributed equally to this work \\ $\triangle$ Corresponding authors: Jia Fan, Tel: +86-21-64037181, E-mail: fan.jia@zs-hospital.sh.cn; Xu-Ting Zhi, Tel: +86-531-82166651, E-mail: zhixuting@hotmail.com; \\ Zhao-Ru Dong, Tel: +86-18560082283, E-mail: dongzhaoru0911@163.com \\ (c) Ivyspring International Publisher. This is an open access article distributed under the terms of the Creative Commons Attribution (CC BY-NC) license \\ (https:// creativecommons.org/licenses/by-nc/4.0/). See http://ivyspring.com/terms for full terms and conditions.
}

Received: 2017.06.04; Accepted: 2017.08.29; Published: 2017.10.17

\begin{abstract}
Surgical resection remains the initial treatment of choice for the majority of early stage hepatocellular carcinoma (HCC) patients. Although the factors that influence the prognosis of postoperative HCC patients have been well elucidated, there are a limited number of simple, objective, and distinct methods for estimating survival for postoperative patients with solitary HCC within the Milan criteria and Child-Pugh (C-P) A cirrhosis. The Albumin-Bilirubin (ALBI) score is a new evidence-based approach to assess liver function. The ALBI score eliminates subjective variables, such as ascites and encephalopathy which are the requirements for the conventional C-P grading system. This study enrolled 654 patients to determine whether the ALBI score can predict the outcomes of postoperative solitary HCC patients within the Milan criteria and C-P A cirrhosis. Our results showed the ALBI score significantly influenced the overall survival and cumulative recurrence rates. Furthermore, the ALBI score was significantly related to the degree of liver cirrhosis and serum Y-glutamyl transpeptidase (GGT) concentration in solitary $\mathrm{HCC}$ cases within the Milan criteria and C-P A cirrhosis. Additionally, the combination of the ALBI score and serum GGT concentration contributed to the prognosis prediction in this cohort. In conclusion, we externally validated the ALBI grade as a novel biomarker to predict prognosis for solitary HCC within the Milan Criteria and C-P A cirrhosis.
\end{abstract}

Key words: Albumin-Bilirubin Score; HCC; Milan Criteria; Child-Pugh A

\section{Introduction}

Hepatocellular carcinoma (HCC) is the most common primary liver malignancy and predominantly occurs in patients with chronic liver diseases, such as chronic hepatitis viral infection [1]. The Barcelona Clinic Liver Cancer (BCLC) staging system is the most widely adopted staging system that provides guidelines for HCC treatment $[2,3]$. According to the BCLC guidelines, patients with a solitary liver tumour or as many as 3 nodules $\leq 3 \mathrm{~cm}$ are classified as having very early or early-stage cancer $[2,4]$. Liver transplantation is considered as the optimal treatment for patients with HCC within the Milan criteria, which includes a solitary HCC of up to $5 \mathrm{~cm}$ in size or of up to 3 tumours $\leq 3 \mathrm{~cm}$ [4]. The shortage of available organs and long waiting times prohibit clinical application of liver transplantation. Surgical resection remains the initial treatment of choice for most of early stage HCC patients because it 
is likely to yield a better prognosis than radiofrequency ablation (RFA) treatment $[5,6]$. Previous studies have elucidated the factors that influence the prognosis of postoperative HCC patients [7-11].

The current prognostic models for HCC include parameters related to $\mathrm{HCC}$, such as the tumour diameter, tumour number, presence of vascular invasion, and extent of tumour differentiation and lymph node metastasis [7, 8]. Previous studies have reported that there is a significantly different prognosis between early HCC patients within the Milan criteria and cases beyond this criterion $[9,10]$. However, the factors that influence the prognosis of HCC patients within the Milan criteria are poorly understood.

Underlying liver function is another significant factor affecting the survival of HCC patients. Liver function is frequently assessed according to the Child-Pugh (C-P) system in HCC patients as a preoperative evaluation of the safety of hepatectomy [9-11]. The C-P system is based on a score derived from the following parameters: conventional liver function tests, degree of hepatic encephalopathy, and extent of ascites. However, this system has several limitations which have been reported by previous references [12, 13]. The Albumin-Bilirubin (ALBI) score is a new evidence-based approach that is used to assess liver function in HCC patients [13]. In this new model, subjective variables, such as ascites and encephalopathy which are the requirements for the conventional C-P grade system, are eliminated. The degree of accuracy achieved by the ALBI grade is considered equal to the accuracy obtained with the conventional C-P grade [13]. We performed this retrospective study to assess the value of the ALBI score for prognosis predictions of solitary HCC cases within the Milan criteria and C-P A cirrhosis because there is confusion regarding live functional assessments and there are no predictive measures for the prognosis of patients with solitary HCC within the Milan criteria.

\section{Patients and Methods}

\section{Patients}

We obtained approval for this retrospective study from the ethical committee of Zhongshan Hospital, Fudan University and Qilu Hospital, Shandong University. The study was performed in accordance with the approved guidelines and written informed consent for the use of the resected samples and clinical data. The study enrolled patients with newly diagnosed HCC within BCLC the early stages who received surgical resection at Zhongshan
Hospital, Fudan University between Jan. 2005 and Dec. 2010, or at Qilu Hospital, Shandong University between Jan. 2009 and Dec. 2011.

The study inclusion criteria included the following: 1) primary solitary HCC within the Milan criteria; 2) curative resection of tumour lesions; 3) no lymph node involvement, no vascular invasion, and no distant metastasis; 4) cirrhosis confirmed by postoperative pathology; and 5) preoperative liver function assessed as the C-P A stage. Follow-up, diagnosis, and treatment of recurrent tumours were performed as described in our previous studies $[2,14$, 15].

The clinicopathological factors potentially related to tumour recurrence and overall survival (OS) were selected based on our previous studies and included the following: gender, age, cirrhosis, the presence of a tumour capsule, tumour diameter, differentiation of tumour cells, and preoperative laboratory values which included the following: serum alanine aminotransferase concentration (ALT $\leq 75 \mathrm{U} / \mathrm{L}$ or $>75 \mathrm{U} / \mathrm{L}$ ), serum a-fetoprotein concentration (AFP $\leq 400 \mathrm{ng} / \mathrm{mL}$ or $>400 \mathrm{ng} / \mathrm{mL}$ ), and serum $\gamma$-glutamyl transpeptidase concentration (GGT $\leq 50 \mathrm{U} / \mathrm{L}$ or $>50 \mathrm{U} / \mathrm{L}$ ). If multiple preoperative serum values were available, the values collected closest to the time of resection were analysed $[2,14,15]$.

\section{Statistical methods}

The continuous variables are expressed as the mean ( \pm sd) or median (range). The categorical variables were compared with the $\chi^{2}$ test or Fisher's exact test. All P-values $<0.05$ were considered to be significant. A univariate survival analysis was performed using the Kaplan-Meier method, and the difference between groups was analysed with the log-rank test. The relative prognostic significance of the variables in predicting the OS and disease-free survival (DFS) was assessed by univariate and multivariate Cox proportional hazards regression models. All statistical analyses of the data were performed using SPSS 21 for Windows (SPSS, Chicago, IL).

\section{Results}

\section{Patients}

This study enrolled 654 HCC patients who met the inclusion criteria and underwent hepatectomy at Zhongshan Hospital, Fudan University between Jan. 2005 and Dec. 2010, or at Qilu Hospital, Shandong University between Jan. 2009 and Dec. 2011. There were $606(92.66 \%)$ patients who were positive for the hepatitis surface B antigen alone and $4(0.61 \%)$ patients were positive for the hepatitis $\mathrm{C}$ antibody alone. Additionally, $7(1.07 \%)$ patients were infected 
with both hepatitis B virus (HBV) and hepatitis C virus (HCV).

The ALBI score of every patient was calculated based on the levels of haemobilirubin and albumin. The equation of the ALBI score for the linear predictor was as follows: linear predictor $=\left(\log _{10}\right.$ bilirubin $\times 0.66)+$ (albumin $\times-0.085$ ), where bilirubin is in $\mu \mathrm{mol} / \mathrm{L}$ and albumin in $\mathrm{g} / \mathrm{L}$ [13]. Patients were divided into two groups according to previously described cut-offs: ALBI grade I $(\leq-2.60)$ and ALBI grade II (>-2.60) [13]. Our study included 477 patients in the ALBI grade I cohort and 177 patients in the ALBI grade II cohort.

There were 5 patients in the ALBI grade I group with both HBV and HCV infections. There were also 439 patients and 3 patients with $\mathrm{HBV}$ or $\mathrm{HCV}$ infection, respectively. Of the $201(42.14 \%)$ patients with recurrent HCC in the ALBI grade I cohort, there were 65 patients who had additional surgery and 7 patients received RFA. There were also 77 patients who received transcatheter arterial chemoembolization (TACE) and/or percutaneous ethanol injection (PEI) and 12 patients received radiotherapy.

There were 2 patients in the ALBI grade II group with both HBV and HCV infections. There were 167 patients and 1 patient with $\mathrm{HBV}$ or $\mathrm{HCV}$ infections, respectively. Of the $99(55.93 \%)$ patients with recurrent HCC in the ALBI grade II cohort, 24 patients had additional surgery and 7 patients received RFA. There were also 24 patients who received TACE and/or PEI. Additionally, 2 patients received radiotherapy and 2 patients received systemic chemotherapy.

\section{Follow-up}

The deadline for follow-up was December 2014. The 1-, 2-, and 5-year OS rates were 94.53\%, 88.40\% and $68.42 \%$ in the ALBI grade I group, respectively. The survival results were significantly different from the OS rates in the ALBI grade II group, which were $92.08 \%, 82.42 \%$ and $56.07 \%$, respectively ( $\chi 2=10.938, p$ $=0.001)($ Figure $1 \mathrm{~A})$.

The 1-, 2-, and 5-year cumulative incidences of intrahepatic recurrence in the ALBI grade I group were $13.90 \%, 28.33 \%$, and $45.33 \%$, respectively. The corresponding values for the ALBI grade II group were $23.92 \%, 37.08 \%$, and $57.28 \%$, respectively. The ALBI grade I group had significantly different cumulative incidences of intrahepatic recurrence compared to the ALBI grade II group ( $x^{2}=9.310$, $p=0.002)$ (Figure 1B).

\section{Multivariate analyses of factors that significantly influenced the OS and DFS for solitary HCC patients within the Milan criteria and C-P A cirrhosis}

The very early stage HCC cases in the BCLC staging system were defined as a solitary lesion $\leq 2$ $\mathrm{cm}$ (without macrovascular invasion or extrahepatic spread) and preserved liver function [4]. Therefore, we used $2 \mathrm{~cm}$ as the cut-off value of the tumour diameter.

The results of the univariate analysis revealed the factors that significantly influenced tumour OS were the ALBI score, cirrhosis, serum GGT concentration and tumour differentiation. Multivariate analysis indicated that ALBI score (hazard ratio, 1.359; 95\% CI, 1.026-1.800; $p=0.033$ ), serum GGT concentration (hazard ratio, 1.685; 95\% CI, 1.277-2.223; $p<0.001$ ), cirrhosis (hazard ratio, 1.449; 95\% CI, 1.089-1.928; $p=0.011)$, and tumour differentiation (hazard ratio, 1.426; 95\% CI, 1.036-1.962; $p=0.029)$ were also significant factors for OS (Table 1).

The univariate analysis results revealed the factors that significantly
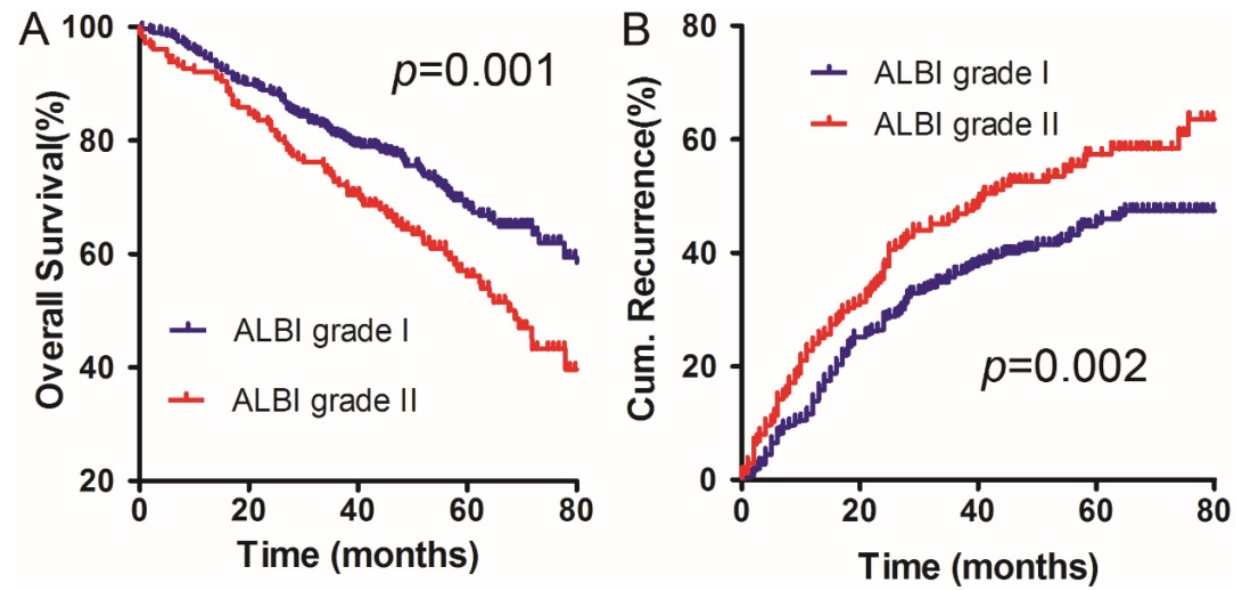

Figure 1. OS (A) and DFS (B) curves for the ALBI grade I group and the ALBI grade Il group in solitary HCC patients within Milan criteria and C-P A cirrhosis. influenced tumour DFS were the ALBI score, sex, serum GGT concentration, and cirrhosis. In the multivariate analysis, the ALBI score (hazard ratio, 1.341; $\quad 95 \% \quad$ CI, 1.047-1.718; $p=0.020)$ and serum GGT concentration (hazard ratio, $1.332 ; 95 \% \mathrm{CI}$, 1.052-1.686; $\quad p=0.017$ ) were also significant factors for DFS (Table 2). 
Table 1. Univariate and multivariate analysis of prognostic factors of OS for the patients with solitary HCC within the Milan criteria and C-P A cirrhosis.

\begin{tabular}{|c|c|c|c|c|}
\hline \multirow[t]{2}{*}{ Variable } & \multicolumn{2}{|l|}{ Univariate } & \multicolumn{2}{|l|}{ Multivariate } \\
\hline & $\begin{array}{l}x^{2} \\
\text { value(Log-rank) }\end{array}$ & $\begin{array}{l}p \\
\text { value }\end{array}$ & $\mathrm{HR}(95 \% \mathrm{Cl})$ & $\begin{array}{l}p \\
\text { value }\end{array}$ \\
\hline Sex (male/female) & 0.966 & 0.326 & - & n.a. \\
\hline Age ( $\leq 52$ vs $>52$ years) & 1.079 & 0.299 & - & n.a. \\
\hline ALBI score & 10.938 & 0.001 & $1.026-1.800$ & 0.033 \\
\hline $\operatorname{ALT}(\leq 75$ vs $>75 \mathrm{U} / \mathrm{L})$ & 0.264 & 0.607 & - & n.a. \\
\hline $\operatorname{AFP}(\leq 400$ vs $>400 \mathrm{ng} / \mathrm{ml})$ & 1.702 & 0.192 & - & n.a. \\
\hline GGT ( $\leq 50 \mathrm{U} / \mathrm{L}$ vs $>50 \mathrm{U} / \mathrm{L})$ & 20.667 & $<0.001$ & $1.277-2.223$ & $<0.001$ \\
\hline Cirrhosis (mild/ severe) & 13.201 & $<0.001$ & $1.089-1.928$ & 0.011 \\
\hline Tumor diameter $(\leq 2 \mathrm{vs}>2 \mathrm{~cm})$ & 0.545 & 0.460 & - & n.a. \\
\hline $\begin{array}{l}\text { Tumor capsule (positive vs } \\
\text { negative) }\end{array}$ & 0.170 & 0.680 & - & n.a. \\
\hline $\begin{array}{l}\text { Tumor differentiation (III/IV vs } \\
\text { I/II) }\end{array}$ & 4.483 & 0.034 & $1.036-1.962$ & 0.029 \\
\hline
\end{tabular}

n.a. not applicable.

\section{Relationship between the ALBI score and clinicopathological parameters of solitary HCC within Milan criteria and C-P A cirrhosis}

We also examined the correlations between the ALBI score and clinicopathological parameters of this patient cohort. We found that the ALBI score of the patients with solitary HCC within Milan criteria and C-P A cirrhosis was significantly related to the degree of liver cirrhosis $(p<0.001)$ and serum GGT concentration $(p<0.001)$. This finding was not related to sex, age, serum ALT concentration, serum AFP concentration, or degree of tumour cell differentiation. In our cohort, HCC patients with severe cirrhosis had significantly higher ALBI scores (stage II) (Table 3).

\section{The combination of the ALBI score and serum GGT concentration for predicting outcomes after hepatic resection for solitary HCC within the Milan criteria and C-P A cirrhosis}

The ALBI score and serum GGT concentration are simple and practicable indicators that significantly influenced the OS and DFS in present cohort. Thus, we further developed a model to predict the prognosis of patients with solitary HCC within the Milan criteria and C-P A cirrhosis. Our results revealed that HCC patients with higher serum GGT levels had a worse prognosis for both OS and cumulative recurrence rates than the low GGT group. This finding was applied to both the ALBI I subgroup $(p=0.001, p=0.042$, respectively, Figure 2A, B) and ALBI II subgroup $(p=0.022, p=0.020$, respectively, Figure $2 \mathrm{C}, \mathrm{D})$. The results suggest that a combination of the ALBI score and serum GGT concentration can more accurately predict the outcomes for patients with solitary HCC within the Milan criteria and C-P A cirrhosis.
Table 2. Univariate and multivariate analysis of prognostic factors of DFS for the patients with solitary HCC within the Milan criteria and C-P A cirrhosis.

\begin{tabular}{|c|c|c|c|c|}
\hline \multirow[t]{2}{*}{ Variable } & \multicolumn{2}{|l|}{ Univariate } & \multicolumn{2}{|c|}{ Multivariate } \\
\hline & $\begin{array}{l}\chi^{2} \\
\text { value(Log-rank) }\end{array}$ & $\begin{array}{l}p \\
\text { value }\end{array}$ & $\begin{array}{l}\mathrm{HR}(95 \% \\
\mathrm{Cl})\end{array}$ & $\begin{array}{l}p \\
\text { value }\end{array}$ \\
\hline Sex (male/female) & 5.134 & 0.023 & $0.989-1.926$ & 0.058 \\
\hline Age ( $\leq 52$ vs $>52$ years) & 0.346 & 0.557 & - & n.a. \\
\hline ALBI score & 9.310 & 0.002 & $1.047-1.718$ & 0.020 \\
\hline $\operatorname{ALT}(\leq 75$ vs $>75 \mathrm{U} / \mathrm{L})$ & 0.040 & 0.842 & - & n.a. \\
\hline $\operatorname{AFP}(\leq 400$ vs $>400 \mathrm{ng} / \mathrm{ml})$ & 1.857 & 0.173 & - & n.a. \\
\hline GGT $(\leq 50 \mathrm{U} / \mathrm{L}$ vs $>50 \mathrm{U} / \mathrm{L})$ & 11.610 & 0.001 & $1.052-1.686$ & 0.017 \\
\hline Cirrhosis (mild/ severe) & 5.181 & 0.023 & $0.923-1.491$ & 0.191 \\
\hline Tumor diameter $(\leq 2 \mathrm{vs}>2 \mathrm{~cm}$ ) & 1.817 & 0.178 & - & n.a. \\
\hline $\begin{array}{l}\text { Tumor capsule (positive vs } \\
\text { negative) }\end{array}$ & 2.589 & 0.108 & - & n.a. \\
\hline $\begin{array}{l}\text { Tumor differentiation (III/IV vs } \\
\text { I/II) }\end{array}$ & 2.043 & 0.153 & - & n.a. \\
\hline
\end{tabular}

Table 3. Clinical features for the patients with solitary HCC within the Milan criteria and C-P A cirrhosis.

\begin{tabular}{|c|c|c|c|}
\hline Characteristics & $\begin{array}{l}\text { ALBI score I ( } \mathrm{n} \\
=477)\end{array}$ & $\begin{array}{l}\text { ALBI score II } \\
(\mathrm{n}=177)\end{array}$ & $p$ value \\
\hline Sex (male/female) & $395 / 82$ & $142 / 35$ & 0.444 \\
\hline Age $(\leq 52 />52$ years $)$ & $247 / 230$ & $83 / 94$ & 0.267 \\
\hline \multicolumn{4}{|l|}{$\operatorname{ALT}(\mathrm{U} / \mathrm{L})$} \\
\hline$\leq 75$ & 410 & 147 & 0.353 \\
\hline$>75$ & 67 & 30 & \\
\hline \multicolumn{4}{|l|}{$\operatorname{AFP}(\mathrm{ng} / \mathrm{ml})$} \\
\hline$\leq 400$ & 359 & 127 & 0.361 \\
\hline$>400$ & 118 & 50 & \\
\hline \multicolumn{4}{|l|}{ GGT(U/L) } \\
\hline$\leq 50$ & 273 & 74 & $<0.001$ \\
\hline$>50$ & 204 & 103 & \\
\hline \multicolumn{4}{|l|}{ Tumor diameter (cm) } \\
\hline$\leq 2$ & 119 & 54 & 0.152 \\
\hline$>2$ & 358 & 123 & \\
\hline $\begin{array}{l}\text { Tumor capsule (positive vs } \\
\text { negative) }\end{array}$ & $277 / 200$ & $104 / 73$ & 0.874 \\
\hline $\begin{array}{l}\text { Tumor differentiation (III/IV } \\
\text { vs I/II) }\end{array}$ & $388 / 89$ & $141 / 36$ & 0.627 \\
\hline Cirrhosis (mild/ severe) & $239 / 238$ & $53 / 124$ & $<0.001$ \\
\hline
\end{tabular}

\section{Discussion}

Cirrhosis-related liver dysfunction is a major cause of death in HCC patients [16, 17]. Clinically, patients with liver dysfunction rarely display evidence of significant coagulopathy, refractory ascites, or encephalopathy and it is difficult to evaluate preserved liver function. Liver function is currently graded according to the C-P system, which relies on individual parameters that are scored based on arbitrarily defined, predetermined cut-off points.

The new ALBI approach is an alternative measure of liver function based solely on albumin and bilirubin, which can avoid inter-observer variation and may highlight distinct subgroups within C-P A cirrhosis. Several studies have analysed the prognostic value of the ALBI score in postoperative 
HCC patients and have proposed its integration with routine prognostic models, including the BCLC [18-20]. Pinato and colleagues used a stage-stratified approach to investigate the prognostic ability of the ALBI score in both curative and palliative settings. The results demonstrated an adequate and clinically meaningful stratifying potential for the score across HCC BCLC stages [18]. Ma et al. also demonstrated the ALBI grade predicted prognosis after curative resection of patients with early HCC [19]. However, eligible HCC patients were enrolled in the studies irrespective of tumour diameters and cirrhosis. These factors could have influenced the survival outcomes of HCC patients [9-11].

In contrast to previous reports, our study analysed the role of the ALBI score in predicting outcomes for postoperative solitary $\mathrm{HCC} \leq 5 \mathrm{~cm}$ and with C-P A cirrhosis. Our results suggested that the ALBI score is significantly related to the degree of liver cirrhosis and is an independent risk factor that is associated with the OS and DFS. Importantly, tumour diameter did not affect the prognosis of patients in this cohort. The ALBI score is superior to the C-P system in predicting the prognosis of patients with solitary HCC within the Milan criteria and C-P A
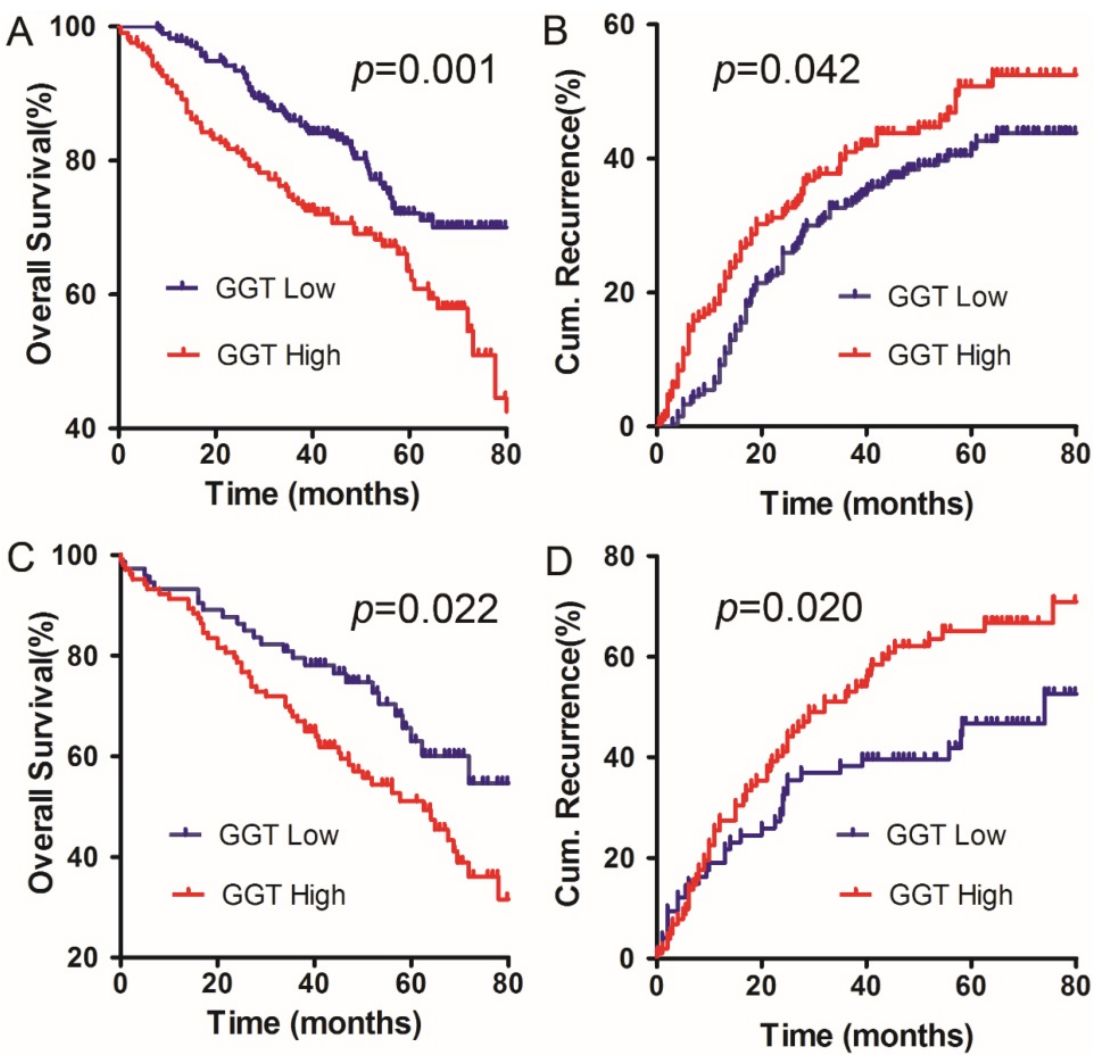

Figure 2. The combination of ALBI score and serum GGT concentration for predicting the prognosis in our cohort. A and B: the OS and DFS curves for patients in ALBI grade I. C and D: the OS and DFS curves for patients in ALBI grade II. cirrhosis.

GGT is a cell-surface enzyme that hydrolyses the gamma-glutamyl bond of extracellular reduced and oxidized glutathione. This initiates cleavage into glutamate, cysteine, and glycine [21, 22]. Several studies have reported that GGT is involved in tumour development, progression, and chemotherapy resistance by releasing reactive oxygen species, controlling of $\mathrm{CpG}$ island methylation, inducing DNA damage and genome instability [21-23]. Clinical studies have shown that increased serum GGT levels are independently associated with the risk of several solid malignancies and always serve as an important prognostic marker for worse OS [24-25]. The predictive value of GGT for HCC patients was shown in cases that underwent hepatectomy and in cases with unresectable HCC that received TACE or chemotherapy [26-28].

We postulated that the ALBI score was significantly related to the serum GGT concentration in solitary HCC within the Milan criteria and C-P A cirrhosis. We found that both the ALBI score and serum GGT concentration could be used as independent prognostic factors for HCC in our cohort. More importantly, HCC patients with a high level of serum GGT had a worse OS and cumulative recurrence rates in the ALBI I and II groups. Therefore, the combination of the ALBI score and serum GGT concentration can be used to identify a population with solitary HCC within the Milan criteria and C-P A cirrhosis that is vulnerable to intrahepatic recurrence.

In our cohort, there were considerable patients with recurrent HCC received subsequent treatments including the following: surgery, RFA, TACE or/and PEI, radiotherapy, and systemic chemotherapy. These treatments may have an important role on overall survival.

In conclusion, our study demonstrated that the ALBI score is a significant prognostic factor correlated with the degree of liver cirrhosis for patients with solitary HCC within the Milan criteria and C-P A cirrhosis. The combination of the ALBI score and serum GGT 
concentration could be used to screen HCC populations within our criteria and determine who would be vulnerable to postoperative intrahepatic recurrence.

\section{Abbreviations}

HCC: Hepatocellular carcinoma; ALBI: Albumin-Bilirubin; C-P: Child-Pugh; RFA: radiofrequency ablation; $\mathrm{HBV}$ : hepatitis $\mathrm{B}$ virus; $\mathrm{HCV}$ : hepatitis $C$ virus; OS: overall survival; DFS: disease-free survival; TACE: transcatheter arterial chemoembolization; PEI: percutaneous ethanol injection; ALT: alanine aminotransferase; AFP: a-fetoprotein; GGT: y-glutamyl transpeptidase.

\section{Acknowledgements}

This study was supported by the National Key Sci-Tech Project (2012ZX10002011-002), the National Natural Science Foundation of China (81602548, 81500122), the Natural Science Foundation of Shandong Province (ZR2016HM06, ZR2014HQ047), and Taishan Scholars Program for Young Expert of Shandong Province (No.tsqn 20161064).

\section{Competing Interests}

The authors have declared that no competing interest exists.

\section{References}

1. Balogh J, Victor D 3rd, Asham EH, et al. Hepatocellular carcinoma: a review. J Hepatocell Carcinoma, 2016, 3:41-53.

2. Dong $\mathrm{ZR}$, Zhang $\mathrm{PF}$, Wang $\mathrm{CH}$, et al. Postoperative adjuvant transcatheter arterial chemoembolization for resectable multiple hepatocellular carcinoma beyond the Milan criteria: a retrospective analysis. Am J Cancer Res, 2015, 5 (1): 450-457.

3. Moore A, Cohen-Naftaly M, Benjaminov O, et al. Radiotherapy and Sorafenib in the Management of Patients with Hepatocellular Carcinoma Have Led to Improved Survival: A Single Center Experience. J Cancer, 2016, 7(8): 883-889.

4. Bruix J, Reig M, Sherman M. Evidence-Based Diagnosis, Staging, and Treatment of Patients With Hepatocellular Carcinoma. Gastroenterology, 2016, 150(4): 835-853.

5. Wang $\mathrm{JH}$, Wang $\mathrm{CC}$, Hung $\mathrm{CH}$, et al. Survival comparison between surgical resection and radiofrequency ablation for patients in BCLC very early/early stage hepatocellular carcinoma. J Hepatol, 2012, 56(2): 412-418.

6. Gory I, Fink M, Bell S, et al. Radiofrequency ablation versus resection for the treatment of early stage hepatocellular carcinoma: a multicenter Australian study. Scand J Gastroenterol, 2015, 50(5): 567-576.

7. Wang XP, Mao MJ, He ZL, et al. A retrospective discussion of the prognostic value of combining prothrombin time(PT) and fibrinogen(Fbg) in patients with Hepatocellular carcinoma. J Cancer, 2017, 8(11): 2079-2087.

8. Zhao H, Chen $\mathrm{C}, \mathrm{Fu} \mathrm{X}$, et al. Prognostic value of a novel risk classification of microvascular invasion in patients with hepatocellular carcinoma after resection. Oncotarget, 2017, 8(3): 5474-5486.

9. Zhang TT, Zhao XQ, Liu Z, et al. Factors affecting the recurrence and survival of hepatocellular carcinoma after hepatectomy: a retrospective study of 601 Chinese patients. Clin Transl Oncol, 2016, 18(8): 831-840.

10. Hoffmann K, Müller-Bütow V, Franz C, et al. Factors predictive of survival after stapler hepatectomy of hepatocellular carcinoma: a multivariate, single-center analysis. Anticancer Res, 2014, 34(2): 767-776.

11. Nishikawa $H$, Kita $R$, Kimura $T$, et al. Hyponatremia in hepatocellular carcinoma complicating with cirrhosis. J Cancer, 2015, 6(5): 482-489.

12. Durand F, Valla D. Assessment of prognosis of cirrhosis. Semin Liver Dis, 2008, 28(1): 110-122.

13. Johnson PJ, Berhane S, Kagebayashi C, et al. Assessment of liver function in patients with hepatocellular carcinoma: a new evidence-based approach-the ALBI grade. J Clin Oncol, 2015, 33(6): 550-558.

14. Dong ZR, Zhang C, Cai JB, et al. Role of 5-hydroxymethylcytosine level in diagnosis and prognosis prediction of intrahepatic cholangiocarcinoma. Tumour Biol, 2015, 36(4): 2763-2771.
15. Cai JB, Shi GM, Dong ZR, et al. Ubiquitin-specific protease 7 accelerates p14(ARF) degradation by deubiquitinating thyroid hormone receptor-interacting protein 12 and promotes hepatocellular carcinoma progression. Hepatology, 2015, 61(5): 1603-1614.

16. Sasaki K, Shindoh J, Margonis GA, et al. Effect of Background Liver Cirrhosis on Outcomes of Hepatectomy for Hepatocellular Carcinoma. JAMA Surg, 2017, 152(3): e165059.

17. Wang Q, Blank S, Fiel MI, et al. The Severity of Liver Fibrosis Influences the Prognostic Value of Inflammation-Based Scores in Hepatitis B-Associated Hepatocellular Carcinoma. Ann Surg Oncol, 2015, 22: S1125-1132.

18. Pinato DJ, Sharma R, Allara E, et al. The ALBI grade provides objective hepatic reserve estimation across each BCLC stage of hepatocellular carcinoma. J Hepatol, 2017, 66(2): 338-346.

19. Ma XL, Zhou JY, Gao XH, et al. Application of the albumin-bilirubin grade for predicting prognosis after curative resection of patients with early-stage hepatocellular carcinoma. Clin Chim Acta, 2016, 462: 15-22.

20. Liu PH, Hsu CY, Hsia CY, et al. ALBI and PALBI grade predict survival for HCC across treatment modalities and BCLC stages in the MELD Era. J Gastroenterol Hepatol, 2017, 32(4): 879-886.

21. Hanigan $\mathrm{MH}$. Gamma-glutamyl transpeptidase: redox regulation and drug resistance. Adv Cancer Res, 2014, 122. 103-141.

22. Kunutsor SK. Gamma-glutamyltransferase-friend or foe within? Liver Int, 2016, 36(12): 1723-1734.

23. Corti A, Duarte TL, Giommarelli C, et al. Membrane gamma-glutamyl transferase activity promotes iron-dependent oxidative DNA damage inmelanoma cells. Mutat Res, 2009, 669(1-2): 112-121.

24. Luo C, Xu B, Fan Y, et al. Preoperative Gamma-Glutamyltransferase Is Associated with Cancer-Specific Survival and Recurrence-Free Survival of Nonmetastatic Renal Cell Carcinoma with Venous Tumor Thrombus. Biomed Res Int, 2017, 2017: 3142926.

25. Grimm $C$, Hofstetter $G$, Aust $S$ et al Association of gammaglutamyltransferase with severity of disease at diagnosis and prognosis of ovarian cancer. Br J Cance, 2013, 109(3): 610-614.

26. Nagasue N, Ono T, Yamanoi A, et al. Prognostic factors and survival after hepatic resection for hepatocellular carcinoma without cirrhosis. Br J Surg, 2001, 88(4): 515-522.

27. Zhang JB, Chen Y, Zhang B, et al. Prognostic significance of serum gamma-glutamyl transferase in patients with intermediatehepatocellular carcinoma treated with transcatheter arterial chemoembolization. Eur J Gastroenterol Hepatol, 2011, 23(9): 787-793.

28. Xia J, Song P, Sun Z, et al. Advances of diagnostic and mechanistic studies of $\gamma$-glutamyl transpeptidase in hepatocellularcarcinoma. Drug Discov Ther, 2016, 10(4): 181-187. 